\title{
Radio-over-Modes for C-RAN Architecture with Smart Optical Resources Assignment
}

\author{
Lorenzo Combi, Andrea Matera, Alberto Gatto, Paola Parolari, Pierpaolo Boffi and Umberto Spagnolini \\ Dipartimento di Elettronica, Informazione e Bioingegneria (DEIB), Politecnico di Milano, Italy \\ E-mails: \{lorenzo.combi, andrea.matera, alberto.gatto, paola.parolari, pierpaolo.boffi, umberto.spagnolini\}@polimi.it
}

\begin{abstract}
In this paper we consider a centralized radio access network (C-RAN) architecture with a fully analog fronthaul link between remote radio heads (RRHs) and baseband units (BBUs) based on the radio over fiber (RoF) paradigm. Mode division multiplexing (MDM) and frequency division multiplexing (FDM) are employed to provide an additional multiplexing signal dimension to meet the huge bandwidth requirements of next generation (5G) wireless mobile systems. The main contribution of the paper is to prove that a smart resource assignment between the radio antennas and the mode/frequency dimensions allows the communication over the RRH-BBU link at rates that are comparable to those achieved by an ideal fronthauling where BBU and RRH are assumed to be co-located, even without any complex and costly optical equalization technique. Validation is on the radio-link capabilities employing multiple antennas to meet the demand for massive MIMO technology.
\end{abstract}

Index Terms-MDM, analog fronthauling, optical resource allocation, massive MIMO, C-RAN

\section{INTRODUCTION}

Next generation (5G) mobile communication networks are expected to cope with the ever-growing demand for high throughput, mobility and low latency which is dramatically changing the paradigm of radio access networks (RANs). The employment of high carrier frequencies, small cells and pervasive antenna deployment seem to be the the only feasible solution to meet such requirements [1].

In this context, centralized RANs (C-RANs) [2] provide several benefits in terms of cost effectiveness and performance optimization through baseband units (BBUs) centralization, allowing to serve a large number of users in the same radio resources by taking advantage of mutual cooperation among BBUs for interference mitigation. The antennas, together with all the RF functionalities, are hosted at the cell sites in the remote radio heads (RRHs), which need to forward the signal to/from the BBUs. Higher bandwidth for the radio signal and massive number of antennas impose strict requirements on the capacity of the fronthaul link between RRHs and BBUs, thus arguing the effectiveness of conventional digital fronthauling based on CPRI protocol [3]. In fact, digital fronthauling is based on the exchange of the in-phase and quadrature (IQ) streaming of the RF signals after digitalization, which leads to a bandwidth expansion (approx. 30x). Several approaches to overcome such limitations have been proposed, such as CPRI compression (bandwidth expansion reduced down to 16-18x) [4] or different functional splits of the RAN [5]. However, the only viable solution appears to be the overtaking

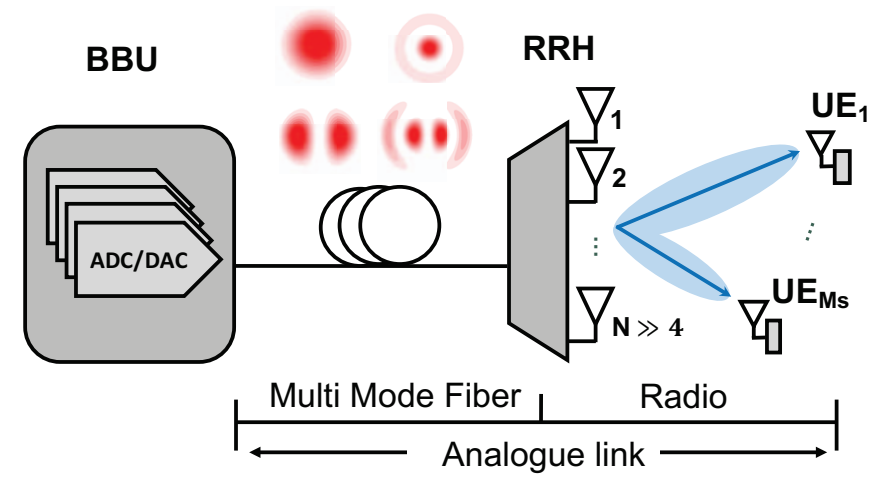

Figure 1: MDM-based C-RAN

of conventional digital fronthauling in favor of a fully ana$\log$ transmission [6]-[9] which enables the reduction of the bandwidth requirements, the latency and the hardware cost, while improving the energy efficiency, since only analog signal relaying is performed at the RRH toward BBU (and viceversa).

One possibility is to deploy an analog fronthaul based on the radio over fiber ( $\mathrm{RoF}$ ) paradigm, due to its capability to carry several Gbit/s $[8,9]$ in terms of equivalent data rate. However, the required bandwidth scales with the number of antennas at the RRH that can be huge if considering the over-6GHz spectrum occupancy (say $\gg 10 \mathrm{GHz}$ ), thus rising the corresponding data-rate up to several tens of Gbit/s for RRH. In this scenario, the huge bandwidth provided by optical link could be even not enough, calling for the need of a new multiplexing dimension. Here we consider mode division multiplexing (MDM) to overcome the bandwidth limitations of RoF architecture by taking advantage of the different propagation modes of light on fiber.

The concept of MDM has old roots [10] and just nowadays it is proposed for longhaul communications thanks to coherent detection and optical-MIMO demultiplexing [11]. However, the application of MDM to C-RAN has relatively short history [12] and not much research efforts have been devoted to its investigation. The main issue is represented by the severe crosstalk among different modes (intermodal crosstalk) introduced by commercial devices available on the market. In fact, the technologies employed in longhaul communications present too high complexity and costs for the access network while the simple employment of all-optical passive modal multiplexer (MUX) and demultiplexer (DEMUX) combined 
with direct detection is not robust enough to cope with intermodal crosstalk.

In this paper we propose a radio over modes (RoM) mobile fronthauling (MFH) exploiting MDM and frequency division multiplexing (FDM) to serve a large number of antennas $(>100)$, supported by low-cost and energy efficient direct detection and all-optical passive (de)multiplexers. In the characterization of the effects of intermodal crosstalk two different technologies are taken into account: photonic lanterns (PLs) [13] and multi-plane light converters (MPLCs) [14]. In particular, we show how to overcome the limitations due to the intermodal crosstalk through a dynamic antennas to mode/frequency mapping, where the optical channels defined in the mode-frequency dimensions are optimally allocated to the signals from/to the RRH antennas, based on the performance metric defined for the radio scenario using the principle that fronthauling should be transparent for RAN.

To have meaningful results, RoM performance evaluation is carried out for a sample scenario in which a beamforming is performed at the BBU to separate the useful uplink signal from interference arising from neighboring hot-spots. The optical channel is assumed to be maximally transparent to the beamformer that is designed based only on statistical knowledge of the radio channel (i.e., not instantaneous).

Contributions: The contributions of the paper are threefold: $i$ ) we propose a RoM MFH exploiting MDM-FDM with direct detection and all-optical passive MUX/DEMUX, ii) we evaluate the performance of the proposed architecture considering a realistic uplink radio scenario with a conventional statistical characterization of the interference, and iii) we show that even without optical-MIMO processing the performance of the proposed architecture, exploiting a novel antennas to modes/frequencies mapping, attains that of the ideal fronthauling.

Notation: Bold upper- and lower-case letters describe matrices and column vectors. $[\mathbf{A}]_{i j}=a_{i j}$ denotes the $i j$ th element of matrix A. Letters $\mathbb{R}, \mathbb{C}$ refer to real and complex numbers, respectively. We denote matrix inversion, transposition and conjugate transposition as $(\cdot)^{-1},(\cdot)^{T},(\cdot)^{H}$. Matrix $\mathbf{I}$ is an identity matrix of appropriate size and $\mathrm{E}[\cdot]$ is the statistical average.

Organization: Section II introduces the system model for the radio scenario and the optical fronthauling. Section III presents the employed beamforming model based on statistical interference characterization, together with the performance metrics adopted in the simulation results in Section IV. Concluding remarks are in Section V.

\section{System Model}

In this paper we consider a C-RAN based on RoF paradigm where optical fronthauling employs MDM and FDM to separate/aggregate signals from/to different antennas. In particular, we focus on beamforming to separate uplink signals from $M_{S}$ users served by a specific cell, with interference coming from $M_{I}$ users in a neighboring cell (downlink would be similar, not covered here). The analog signals received at the RRH

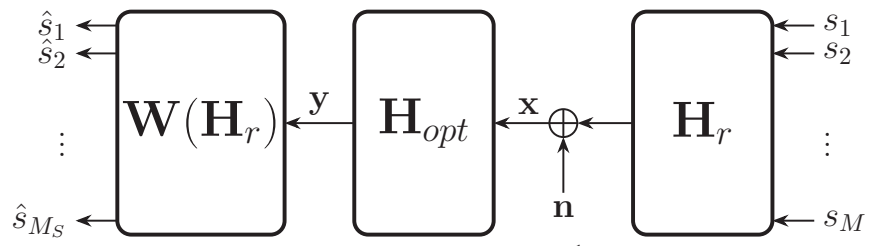

Figure 2: System model, uplink

by $N$ antennas are directly relayed through a multimode fiber (MMF) link towards the BBU, where receiver processing is performed (e.g., beamforming). Therefore the estimated user signal $\hat{\mathrm{s}}$ at the $\mathrm{BBU}$ is described by

$$
\hat{\mathbf{s}}=\mathbf{W H}_{\text {opt }}\left(\mathbf{H}_{r} \mathbf{s}+\mathbf{n}\right),
$$

where the matrices $\mathbf{W}, \mathbf{H}_{o p t}$ and $\mathbf{H}_{r}$ are respectively the beamformer, the optical channel, and the radio channel shown in Fig. 2 and defined in the following sections. Vectors $\mathbf{s}$ and $\mathbf{n}$ contain the user signals and the noise at the antenna array respectively, and optical noise is neglected (or accounted for in the noise vector $\mathbf{n}$ ).

\section{A. Radio scenario}

The signals received by the $N$ antennas at the RRH, arranged in a vector, are:

$$
\mathbf{x}=\mathbf{H}_{r} \mathbf{s}+\mathbf{n}
$$

where $\mathbf{s} \in \mathbb{C}^{M}$ collects the signals with power $\sigma_{s}^{2}$ transmitted by all the $M=M_{S}+M_{I}$ users and assumed to be Gaussian distributed, i.e., $\mathbf{s} \sim \mathcal{C N}\left(\mathbf{0}, \sigma_{s}^{2} \mathbf{I}\right) . \mathbf{H}_{r} \in \mathbb{C}^{N \times M}$ is the wireless channel matrix and $\mathbf{n} \sim \mathcal{C N}\left(\mathbf{0}, \mathbf{R}_{n}\right)$ is the $N \times 1$ noise vector. The wireless channel model is based on the far field approximation and it accounts for the directions of arrival (DoAs) of the signals:

$$
\mathbf{H}_{r}=\left[\begin{array}{llll}
\beta_{1} \mathbf{a}\left(\theta_{1}\right) & \beta_{2} \mathbf{a}\left(\theta_{2}\right) & \cdots & \beta_{M} \mathbf{a}\left(\theta_{M}\right)
\end{array}\right]
$$

where $\beta_{i}^{2}=1 / d_{i}^{2}$ is the path loss attenuation related to the distance $d_{i}$ from the $i$-th user to the array, $\mathbf{a}\left(\theta_{i}\right) \in \mathbb{C}^{N}$ is the steering vector for the direction of arrival (DoA) $\theta_{i}$ of the $i$-th user. Under the assumption of $\lambda / 2$-spaced uniform linear array (ULA) at the RRH, the steering vector for the $i$-th user is:

$$
\mathbf{a}\left(\theta_{i}\right)=\left[\begin{array}{llll}
1 & e^{j \pi \sin \left(\theta_{i}\right)} & \cdots & e^{j \pi(N-1) \sin \left(\theta_{i}\right)}
\end{array}\right]^{T} .
$$

For simplicity, no fading nor multipath propagation are considered but extension would just include a temporal processing. Furthermore, it is here assumed the presence of a single user to be served in the cell of interest $\left(M_{S}=1\right)$. The symbols transmitted by each user (including the interferers) are assumed to be normalized to have unit power $\left(\sigma_{s}^{2}=1\right)$. 


\section{B. MDM-based optical fronthaul with direct detection}

The (analog) radio signal $\mathbf{x}$ received at the $\mathrm{RRH}$ is directly forwarded over a fiber connection without any digitalization. In this paper we propose to employ multimode optical transmission for such fronthaul links and to have the signals from the different antennas multiplexed in the mode-frequency dimension, with simultaneous use of MDM and FDM. Let us first consider a simplified environment in which only MDM is used to aggregate few antennas, and let us consider optical channel matrices measured in literature and described in the following. The signal $\mathbf{y} \in \mathbb{C}^{N}$ received at the BBU can be described as:

$$
\mathbf{y}=\mathbf{H}_{o p t} \mathbf{x}=\mathbf{H}_{o p t}\left(\mathbf{H}_{r} \mathbf{s}+\mathbf{n}\right)
$$

where $\mathbf{H}_{\text {opt }} \in \mathbb{R}^{N \times N}$ represents the multimodal channel model: the main diagonal elements $\left[\mathbf{H}_{o p t}\right]_{i i}$ are the insertion losses and the off-diagonal terms $\left[\mathbf{H}_{o p t}\right]_{i j}$ represent the intermodal coupling coefficients from the $j$-th to the $i$-th mode. A degree of freedom in the system management is given by the possibility to optimize the optical channel assignment, that is the optimal association between each of the $N$ RRH antennas and fiber modes based on end-to-end performance metrics for radio link defined in Section III-B. This can be modeled with an optical channel described by any permutation of a reference optical channel matrix as:

$$
\mathbf{H}_{o p t}=\mathbf{P}^{T} \mathbf{H}_{r e f} \mathbf{P}
$$

where $\mathbf{P}$ is a permutation matrix that is for a reference optical channel matrix $\mathbf{H}_{r e f}$ which is the coupling matrix taken from the literature.

The optical channel assignment is shown in Fig. 3, where the effect of the permutation matrix $\mathbf{P}$ is highlighted.

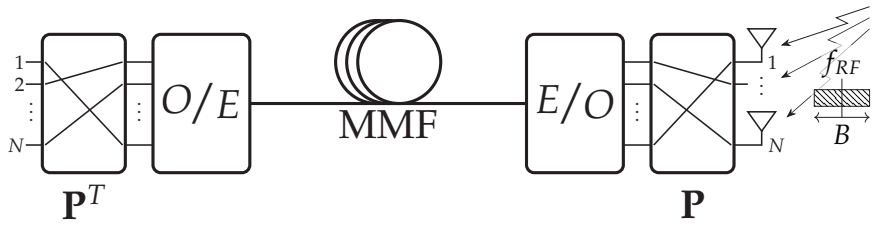

Figure 3: MDM/FDM optical channel assignment

In particular, passive and all-optical devices are considered for mode multiplexing and demultiplexing just to minimize any architectural complexity and to avoid the use of coherent detection and optical-MIMO processing, thus reducing cost and power consumption. Among the different propagation modes, degenerate modes show the same propagation constant but orthogonal states of polarization, therefore in case of direct detection it is impossible to separate them without a suitable polarization control: in this case the pairs of degenerate modes constitute fully-coupled channels that remarkably impair the antennas array capability. Fiber propagation is on weaklycoupled few-mode fibers (FMFs), that show weak intermodal crosstalk, in the order of $-30 \mathrm{~dB} / \mathrm{km}$ for commercial 6-LP FMF allowing the propagation of 4 non-degenerate modes corresponding to 6 spatial modes [15]. Prototype 12-LP FMF (with 7 non-degenerate modes corresponding to 12 spatial modes) is currently a research matter. In a broader temporal horizon, 50- $\mu \mathrm{m}$ graded-index multi-mode fiber (MMF) can support 30 LP modes (55 spatial modes). As far as the mode (de)multiplexing is concerned, the actual crosstalk measurements for (de)multiplexers available on the market or described in literature for out-of-coherence sources is taken into account here, to show the actual feasibility of the proposed RoM architecture. Two different technologies are considered: 6-LPs [16] and 10-LPs [17] non-mode selective PLs - emerging as the most promising and compact multiplexing solution in case of high number of modes and optical-MIMO-assisted demodulation - and MPLC-based solutions allowing commercial MUX/DEMUXs operating up to 10 modes [18].

\section{BEAMFORMING MODEL}

Beamforming, that is performed at the BBU side, is supposed to act on the signals received by the antenna array. This means that beamforming does not act directly on the RRH signal $\mathbf{x}$, but on the signal $\mathbf{y}=\mathbf{H}_{o p t} \mathbf{x}$ received after the optical channel $\mathbf{H}_{o p t}$, which is transparent to the beamforming design.

Minimum variance distortionless (MVDR) beamforming is adopted here to retrieve an estimate of the signal transmitted by the user from the overall signal $\mathbf{y}$ received at the BBU:

$$
\hat{s}=\mathbf{w}_{\mathrm{MVDR}}^{H}\left(\theta_{U E}\right) \mathbf{y}
$$

where $\mathbf{w}_{\text {MVDR }} \in \mathbb{C}^{N}$ is the spatial filter for the DoA of the user, indicated with $\mathrm{w}$ for compactness in the following. MVDR beamforming aims at minimizing the overall mean power after the spatial filtering with the constraint that for the DoA of interest $\theta_{U E}$ the beamforming response has unit-gain (distortionless constraint):

$$
\mathbf{w}=\frac{\mathbf{R}_{y}^{-1} \mathbf{a}\left(\theta_{U E}\right)}{\mathbf{a}^{H}\left(\theta_{U E}\right) \mathbf{R}_{y}^{-1} \mathbf{a}\left(\theta_{U E}\right)} .
$$

The matrix $\mathbf{R}_{y}$ indicates the covariance matrix that contains both the signal of interest and the interference plus noise. Since these contributions can be considered as mutually independent, and assuming the noise at the array to be spatially white, it is:

$$
\mathbf{R}_{y}=\beta_{U E}^{2} \mathbf{a}\left(\theta_{U E}\right) \mathbf{a}^{H}\left(\theta_{U E}\right)+\mathbf{R}_{n+i}
$$

where $\beta_{U E}^{2}$ is the received power at the array from the user of interest; $\mathbf{R}_{n+i}=\mathbf{R}_{I}+\sigma_{n}^{2} \mathbf{I}$ is the interference $\left(\mathbf{R}_{I}\right)$ plus noise $\left(\sigma_{n}^{2} \mathbf{I}\right)$ spatial covariance matrix at the antenna array.

\section{A. Interference statistical model}

It is realistic to assume that, while the DoAs of the users in the served cell are known (or separately estimated), the interference coming from the neighboring cell is available in terms of spatial covariance for the beamforming design at the BBU. Therefore, the covariance matrix for a set of interferers, characterized by a power $\beta_{k}^{2}$ received by the array and a DoA $\theta_{k}$ each, is known only in its expected value: 


$$
\mathbf{R}_{I}=\mathrm{E}\left[\sum_{k=1}^{M_{I}} \beta_{k}^{2} \mathbf{a}\left(\theta_{k}\right) \mathbf{a}\left(\theta_{k}\right)^{H}\right]
$$

where the expectation is taken with respect to the random variables $\left\{\beta_{k}^{2}\right\}_{k=1}^{M_{I}}$ and $\left\{\theta_{k}\right\}_{k=1}^{M_{I}}$ used to describe each interferer. The received power $\beta_{k}^{2}=1 / d_{k}^{2}$ accounts for the path loss as in (3). To obtain the expected value in (10), the first step is to derive the joint probability density function (pdf) $f\left(\beta^{2}, \theta\right)$ of the received power $\beta^{2}$ and DoA $\theta$ for one interferer uniformly distributed over the service area (see Fig. 4).

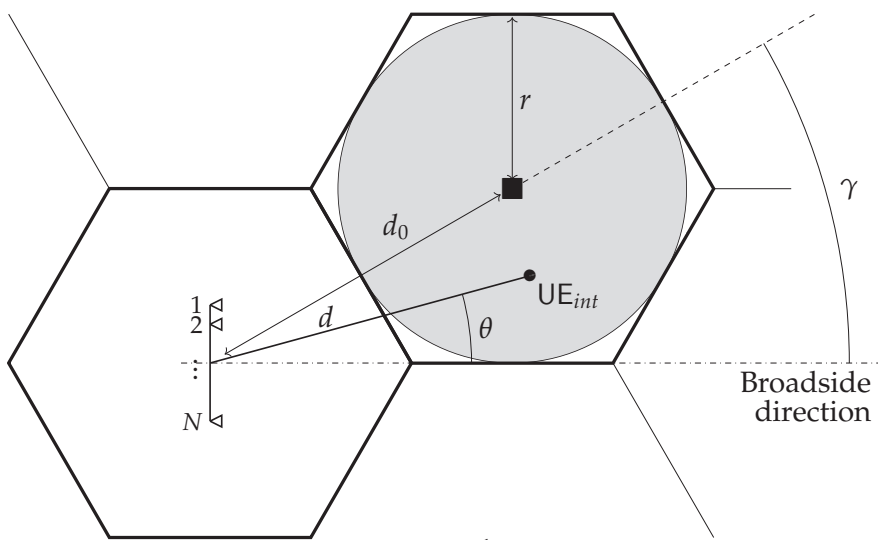

Figure 4: Geometric model for the statistical description of inter-cell interference

Approximately, the interfering cell can be modeled as circular with radius $r$, at distance $d_{0}$ from the array and angular position $\gamma$, and interfering users are uniformly distributed. The joint pdf of received power and DoA is:

$$
f\left(\beta^{2}, \theta\right)=Z \cdot \beta^{-3}\left(d_{\max }(\theta)+d_{\min }(\theta)\right)
$$

where $Z$ is a suitable normalization factor and the pdf is defined for $\theta \in\left[\theta_{\min }, \theta_{\text {max }}\right]$ and $\beta^{2}(\theta) \in$ $\left[1 / d_{\max }(\theta), 1 / d_{\min }(\theta)\right]$. The set $\Theta=\left[\theta_{\min }, \theta_{\max }\right]$ is the support for the DoAs of the interferers, $d_{\min }(\theta)$ and $d_{\max }(\theta)$ are the minimum and maximum distances associated with a specific DoA.

It can be shown (not here for compactness) that the elements of the interference covariance matrix are:

$$
\begin{aligned}
& {\left[\mathbf{R}_{I}\right]_{n, m}=} \\
& 2 M \cdot Z \cdot \int_{\Theta} e^{j \pi(n-m) \sin (\theta)}\left[\frac{d_{\max }^{2}(\theta)-d_{\min }^{2}(\theta)}{d_{\min }(\theta) d_{\max }(\theta)}\right] d \theta
\end{aligned}
$$

where $\Theta=\left[\theta_{\text {min }}, \theta_{\text {max }}\right]$ is the support for the DoAs of the interferers, $d_{\min }(\theta)$ and $d_{\max }(\theta)$ are the minimum and maximum distances associated with a specific DoA and $Z$ is the normalization factor in (11).

\section{B. Performance metrics}

The performance degradation introduced by intermodal crosstalk is evaluated primarily in terms of SINR at the decision variable $\hat{s}$ for the user. SINR after beamforming, in the ideal case of transparent fronthauling, is:

$$
\operatorname{SINR}_{\text {ideal }}\left(\theta_{U E}\right)=\frac{\sigma_{s}^{2}\left|\mathbf{w}^{H} \mathbf{a}\left(\theta_{U E}\right)\right|^{2}}{\mathbf{w}^{H} \mathbf{R}_{n+i} \mathbf{w}} .
$$

For a non-ideal optical channel, the received signal at the beamformer is that of (5), and the optical channel matrix is included in the SINR expression accordingly:

$$
\operatorname{SINR}\left(\theta_{U E}\right)=\frac{\sigma_{s}^{2}\left|\mathbf{w}^{H} \mathbf{H}_{o p t} \mathbf{a}\left(\theta_{U E}\right)\right|^{2}}{\mathbf{w}^{H} \mathbf{H}_{o p t} \mathbf{R}_{n+i} \mathbf{H}_{o p t}^{H} \mathbf{w}}
$$

Performances are evaluated in terms of the wireless channel capacity

$$
C\left(\theta_{U E}\right)=\log _{2}\left(1+\operatorname{SINR}\left(\theta_{U E}\right)\right) .
$$

Moreover, to gain insight into how the system performances scale with the antenna array dimension, an average capacity per user is also evaluated by averaging $C\left(\theta_{U E}\right)$ over $\theta_{U E}$.

\section{Simulation Settings AND Results}

The performances of the proposed MDM/FDM-based fronthaul architecture are evaluated for the following scenario: uplink transmission is considered for a single user $\left(M_{S}=1\right)$, located in the area served by the antenna array $\left(\theta_{\min }=-60^{\circ}\right.$, $\left.\theta_{\max }=60^{\circ}\right)$. The cells have radius $r=500 \mathrm{~m}$ and the interfering cell is at a distance $d_{0}=2 r$, with angular position $\gamma=30^{\circ}$ with respect to the broadside direction of the array. Initially, the number of MDM channels is assumed to be equal to the number of antennas, i.e., no FDM is performed.

First the SINR is evaluated varying the DoA of the user of interest to have a meaningful comparison with the performance offered by beamforming at the RRH (i.e., ideal fronthauling). In Fig. 5, the $\operatorname{SINR}(\theta)$ is evaluated for all the possible permutations $\mathbf{P}$ in (6) of the reference optical channel $\mathbf{H}_{r e f}$ (6 non-degenerate LP modes, PL MUX/DEMUX), highlighting the potential of optimal channel assignment. The SINR degradation experienced around $\theta=30^{\circ}$ is clearly due to the relative positions of antenna array, served user and interfering cell.

The channel capacity $C(\theta)$, corresponding to $\operatorname{SINR}(\theta)$, is then evaluated choosing the best optical channel assignment for each DoA. In Fig. 6, either only non-degenerate modes or all the available spatial modes are exploited in the MDM link. As explained in Section II-B, pairs of degenerate modes result in fully-coupled channels: for this reason the channel matrix, if using all the available modes, presents reduced rank. Results in Fig. 6 confirm that the use of a couple of degenerate modes makes the channel capacity comparable to the one obtained with a number of MDM channels equal to the matrix rank (and hence a lower number of antennas): the green dash-dotted line corresponding to eight MDM channels with two pairs of fullycoupled modes (LP1 $1_{a}-\mathrm{LP} 11_{b}$ and LP2 $\left.1_{a}-\mathrm{LP} 21_{b}\right)$ is close to the solid red line obtained using only the six non-degenerate modes out of the previous eight (LP1 $1_{a+b}$ and LP2 $1_{a+b}$ ). 


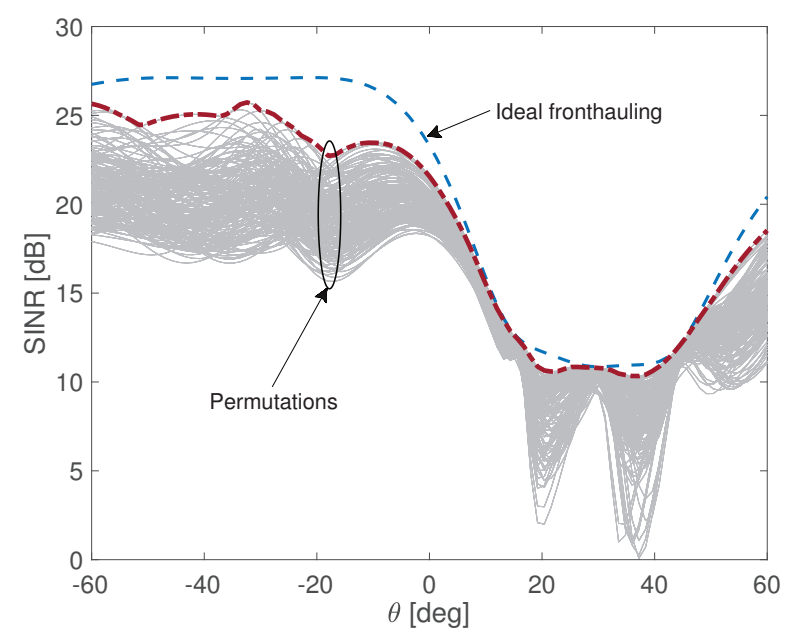

Figure 5: Effect of the optical channel assignment on the SINR

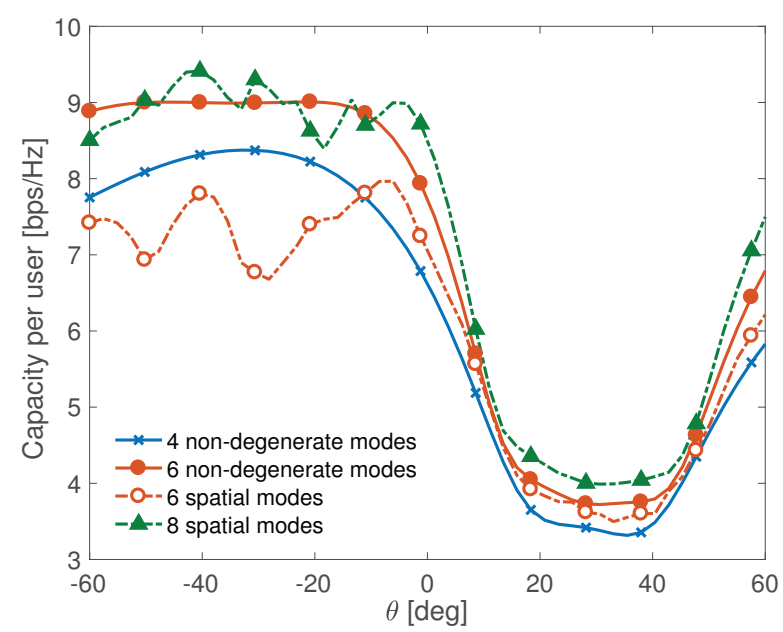

Figure 6: Capacity per user with MPLC MUX/DEMUX vs DoA $\theta_{U E}$ with non-degenerate modes (solid lines) and with spatial modes (dash-dotted lines)

It is therefore mandatory to implement an MDM system based only on non-degenerate modes to take fully advantage of the spatial diversity provided by the RRH antennas. System performances are then evaluated considering only nondegenerate modes: LP01, LP1 $11_{a+b}, \mathrm{LP} 02, \mathrm{LP} 21_{a+b}, \mathrm{LP} 12_{a+b}$ and LP31 $1_{a+b}$. In Fig. 7 the two different technologies considered (MPLCs and PLs) are compared in terms of average capacity per user varying the number of antennas $N$. The solid lines are obtained without FDM, showing far better performances for MPLCs, approaching the ideal curves (dash-dotted) thanks to their higher mode selectivity with respect to PLs. Dashed lines refer to a joint MDM-FDM architecture with PL multiplexing, employing two frequency channels to serve the same number of antennas (i.e., $N / 2$ modes per frequency), providing significant gain with respect to the single-frequency case and approaching the performance of ideal fronthauling.

So far, the average covariance matrix is considered in

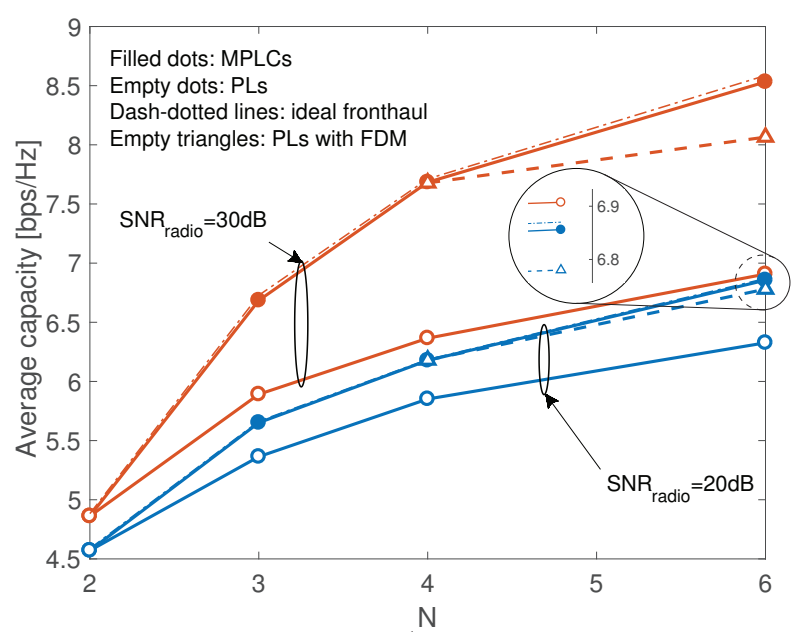

Figure 7: Average capacity vs number of antennas (modes) at 20dB and $30 \mathrm{~dB}$ of radio-link SNR, 10-km fiber propagation

the SINR and in the corresponding capacity. It is however more realistic to assume asynchronous operation of the two neighboring cells in the considered scenario: instead of a fixed number of ever-active $M_{I}$ interferers, the number of simultaneously active interfering users is a random variable, which is modeled as Poisson distributed with average $M_{I}$. In Fig. 8a (shown on the top of next page) the cumulative distribution function $(\mathrm{CDF})$ for the capacity $C\left(\theta_{U E}=-40^{\circ}\right)$ is shown for an average of $M_{I}=6$ interfering users in the neighboring cell and the two considered multiplexing technologies after $1-\mathrm{km}$ fiber propagation, together with the capacity obtained considering the average spatial interference covariance. Fig. 8 b shows the capacity at $5 \%$ outage probability for all DoAs in the same settings.

Although the previous results are obtained for a single user and with a relatively small number of antennas (due to computational issues only), the model can be straightforwardly generalized to serve multiple users with a higher number of antennas. In this setting the size of the optimization problem for the optical resource allocation increases dramatically, following a $N$ ! trend. Thus some more refined optimization technique based on integer programming needs to be developed. Since the optical FDM channels are mutually orthogonal, the overall channel matrix $\mathrm{H}_{o p t}$ is sparse, as crosstalk is only between signal in the same FDM channel, which can be an advantage in terms of optimization efficiency. Moreover, in a realistic scenario multiple users are to be managed inside the cell: the resource allocation optimization needs to be extended to multiobjective optimization, e.g., by considering the weighted sum rate or the minimum rate as objective function.

\section{CONCluding REMARKS}

The implementation of a joint MDM-FDM based fronthauling has been proposed to allow cost-effective and bandwidthefficient connections between massive antenna arrays and centralized BBUs. Direct detection is the key for cost reduction 


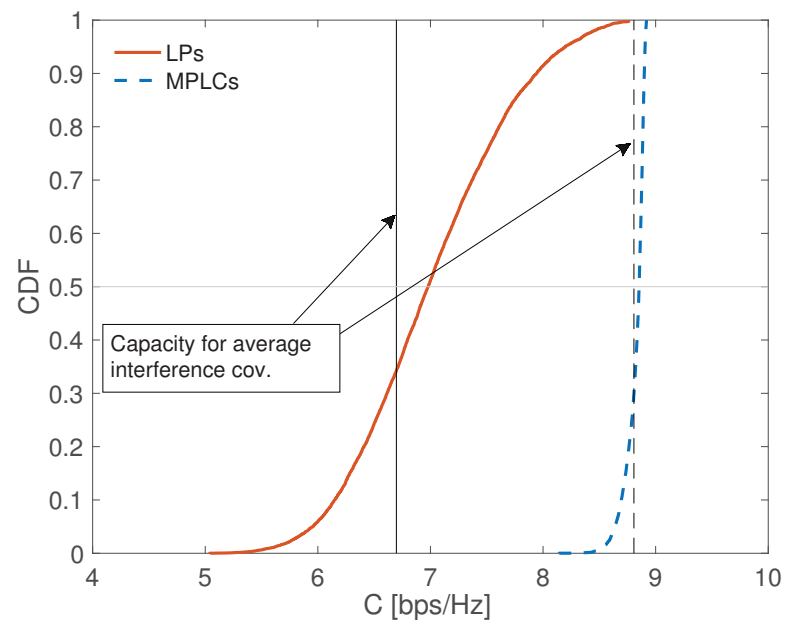

(a) Cumulative distribution function for the capacity $C\left(\theta_{U E}=-40^{\circ}\right)$

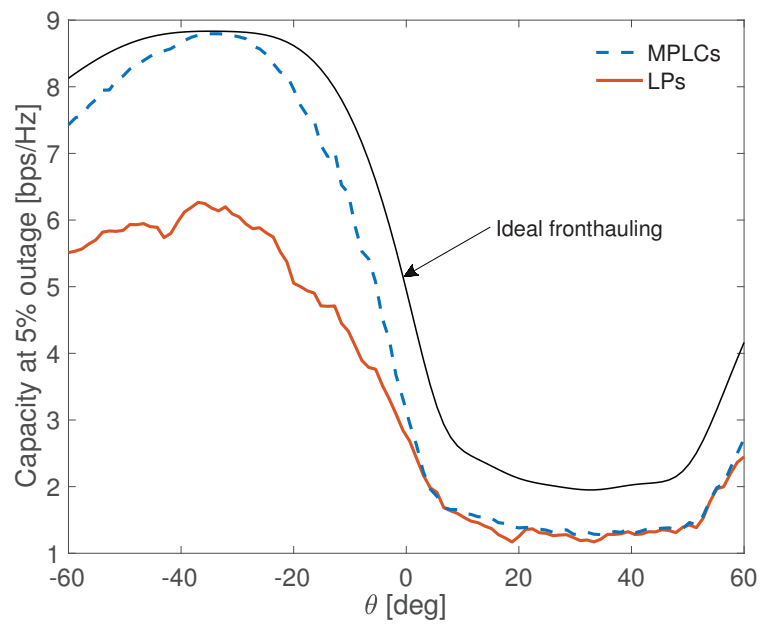

(b) Capacity at $5 \%$ outage probability vs $\theta$

Figure 8: $\mathrm{CDF}$ for $\theta_{U E}=-40^{\circ}$ and capacity at $5 \%$ outage probability, $\mathrm{SNR}_{\text {radio }}=20 \mathrm{~dB}, N=6$ antennas and modes

and energy efficiency, at the price of a reduction in the number of available mode channels due to the presence of degenerate modes. Realistic measured performance of alloptical passive (de)multiplexers as PLs and MPLCs available on the market or described in literature have been taken into account, showing that the employment of degenerate $\mathrm{LPxy}_{a}$ and LPxy ${ }_{b}$ modes (at the same propagation constant) does not assure extra capacity per user with respect to the exploitation of non-degenerate modes only. A statistical interference model has been developed to consider a realistic radio scenario, and MVDR beamforming (transparent to the optical channel) has been considered to evaluate the impact of intermodal crosstalk. Numerical results prove that the use of dynamic assignment for the mode-frequency optical resources allows to overcome the crosstalk limitations and to approach the ideal performances, thus enabling C-RAN based on the joint exploitation MDM-FDM even without conventional solution for intermodal crosstalk mitigation relying on coherent detection and opticalMIMO processing. Further refinement of the optimization technique is needed to extend the results to multiple users and huge antenna arrays, which is left as future research topic.

\section{REFERENCES}

[1] F. Boccardi et al., "Five disruptive technology directions for 5G," IEEE Communications Magazine, vol. 52, no. 2, pp. 74-80, February 2014.

[2] A. Checko et al., "Cloud ran for mobile networks - a technology overview," IEEE Communications Surveys Tutorials, vol. 17, no. 1, pp. 405-426, 2015.

[3] “CPRI Specifications V.6.1 (2014-07-01)," September 2014.

[4] S. Nanba and A. Agata, "A New IQ Data Compression Scheme for Front-haul Link in Centralized RAN," in IEEE PIMRC, London, Sept. 2013.
[5] J. Bartelt et al., "Fronthaul and Backhaul Requirements of Flexibly Centralized Radio Access Networks," IEEE Wireless Communications, Oct. 2015.

[6] J. Gambini and U. Spagnolini, "Wireless over cable for femtocell systems," IEEE Communications Magazine, vol. 51, no. 5, pp. 178-185, May 2013.

[7] L. Combi et al., "Pulse-Width optical modulation for CRAN fronthauling," in IEEE GLOBECOM, San Diego, Dec. 2015.

[8] D.Wake et al., "Radio Over Fiber Link Design for Next Generation Wireless Systems," Journal of Lightwave Technology, vol. 28, no. 16, pp. 2456-2464, 2010.

[9] H. S. Chung et al., "Design of RoF based Mobile Fronthaul Link with Multi-IF Carrier for LTE/LTE-A Signal Transmission," in IEEE APMP, 2014.

[10] S. Berdagué and P. Facq, "Mode division multiplexing in optical fibers," Applied optics, vol. 21, no. 11, pp. 1950-1955, 1982.

[11] N. K. Fontaine et al., "30x30 MIMO transmission over 15 spatial modes," in Optical Fiber Communication Conference Post Deadline Papers. Optical Society of America, 2015, p. Th5C.1.

[12] Y. Chen et al., "Cost effective wavelength reused MDM system for bidirectional mobile fronthaul," Optics Express, vol. 24, no. 20, pp. 22 413-22 422, 2016.

[13] N. K. Fontaine et al., "Evaluation of photonic lanterns for lossless mode-multiplexing," in European Conference and Exhibition on Optical Communication. Optical Society of America, 2012, pp. Th-2.

[14] J.-F. Morizur et al., "Efficient and mode-selective spatial multiplexer based on multi-plane light conversion," in Optical Fiber Communication Conference. Optical Society of America, 2015, pp. W1A-4.

[15] P. Sillard, "Few-mode fibers for space division multiplexing," in Optical Fiber Communication Conference. Optical Society of America, 2016, pp. Th1 J-1.

[16] D. Yu et al., "Mode-dependent characterization of photonic lanterns," Optics letters, vol. 41, no. 10, pp. 2302-2305, 2016.

[17] Velazquez-Benitez et al., "Scaling the fabrication of higher order photonic lanterns using microstructured preforms," 2015.

[18] G. Labroille et al., "Mode selective 10-mode multiplexer based on multiplane light conversion," in Optical Fiber Communication Conference. Optical Society of America, 2016. 